\title{
The relativistically invariant expansion of a scalar function on imaginary Lobachevski space
}

\author{
E. G. Kalnins \\ Centre de Recherches Mathématiques, Université de Montréal, Case Postale 6128, Montréal 101, Québec, \\ Canada \\ (Received 15 September 1972; final revised manuscript received 7 March 1973)
}

\begin{abstract}
Using the previous analysis of Gel'fand and Graev a new relativistically invariant expansion of a scalar function on three-dimensional imaginary Lobachevski space $L_{3}(I)$ is given. The coordinate system used corresponds to the horospherical reduction $S O(3,1) \supset E_{2} \supset S O(2)$ and covers all of $L_{3}(I)$.
\end{abstract}

\section{INTRODUCTION AND SUMMARY}

Explicit relativistically invariant expansions of functions defined on the three transitivity surfaces of the proper Lorentz group in Minkowksi space have been studied to varying degrees in recent years. $1-3$ Of these surfaces explicit expansions on the upper sheet $\mathrm{H}_{2}$ of the double sheeted hyperboloid $4[x, x]=1$ and on the cone $^{5}[\xi, \xi]=0$ have been well developed. 6 (Note: $x$ is a 4-vector in Minkowski space with $[x, x]=x_{0}^{2}-x^{2}$ the usual scalar product.) The explicit expansions on $H_{2}$ and on the cone are based on the expansion formulas due to Gel'fand et al, 7 The invariant expansion of a scalar function $f(x)\left(x \in H_{2}\right)$ is obtained by observing that $H_{2}$ corresponds to a realization of three-dimensional real Lobachevski space $L_{3}(R)$. An invertable horospherical integral transform then associates a function $h(\xi)$ on the cote with each $f(x)$. The invariant expansion of $f(x)$ then reduces to the invariant expansion of $h(\xi)$. The latter expansion is achieved by the decomposition of $h(\xi)$ into homogeneous components.

An analogous geometry and irreducible decomposition of a function $f(x)$ on the single sheeted hyperboloid $H_{1}$, with equation $[x, x]=-1$, has also been given in Ref. 7 . The geometry of $H_{1}$ corresponds to a realization of imaginary Lobachevski space $L_{3}(I)$ and identifies diametrically opposed points [so that $f(x)=f(-x)$ ]. The irreducible decomposition on $H_{1}$ differs from that on $\mathrm{H}_{2}$ in that it contains a discrete spectrum as well as the usual continuous spectrum.

Previously there has been (to the author's knowledge) one paper by Kuznetzov and Smorodinski 8 which has considered an explicit complete set of functions on $H_{1}$ realized as $L_{3}(I)$. This analysis uses the results of Ref. 7 only insofar as they consider a parametrization of $x \in H_{1}$ for which the discrete spectrum term is not necessary. [More specifically, they choose a coordinate system which only parametrizes points at a real distance from $x=(0,0,0,1)$.] Verdiev, ${ }^{9}$ on the other hand, has given his attention to finding an explicit set of complete functions with spin on $H_{1}$. There are some shortcomings in Verdiev's work in that the continuous spectrum expansion functions have not been normalized and the method used to obtain the normalized discrete spectrum expansion functions needs some explanation. Zmuidzinas $^{2}$ has given a complete account of the expansion of a scalar function defined on $H_{1}$ using the eigenfunction expansion methods of Titchmarsh. ${ }^{10}$ This analysis has been done in the canonical group reduction $S O(3,1) \supset S O(3) \supset S O(2)$ or $S$ system. Limic et al . ${ }^{3}$ have treated the general problem of the expansion of square integrable functions defined on the transitivity surfaces of $S O(p, q)$ in the canonical group reduction and hence include the results of Zmuidzinas as a special case.

In this paper we examine the expansion of a square integrable function defined on $L_{3}(I)$ in the noncanonical group reduction $S O(3,1) \supset E(2) \supset S O(2)$ or horospherical system. This expansion is new and serves to illustrate how the analysis of Gel'fand and Graev should be treated to yield the correct expansion formulas. There is only one other group reduction which parametrizes all of $L_{3}(I)$ (apart from the group reduction $S O(3,1) \supset E(2) \supset$ $T_{1} \otimes T_{2}$, which differs little from the horospherical system). This is the $S$ system. We do not however give this expansion here as it differs little from the results of Zmuidzinas and Limic et al.

The study of the horospherical system group reduction of $S O(3,1)$ has received attention previously in application to particle physics 11,12 and is also of intrinsic group theoretical interest.

The content of this paper is arranged as follows. In Sec. 1 we collect the pertinent facts concerning the Gel'fand-Graev analysis on $L_{3}(I)$. In Sec. 2 we give the horospherical system expansion.

\section{THE HARMONIC ANALYSIS OF A SCALAR FUNCTION ON $L_{3}(/)$}

The central problem here is the decomposition of the representation

$$
\left[T_{g} f\right](x)=f(x g), \quad x \in L_{3}(I)
$$

into components which transform according to unitary irreducible representations (UIRs) of the proper Lorentz group $S O(3,1)$. The Gel'fand-Graev transform on $L_{3}(I)$ invertably maps $f(x)$ into a pair of functions $h(\xi)$ and $\phi(\xi, b)$. The function $h(\xi)$ gives the representation

$$
\left[Q_{g} h\right](\xi)=h(\xi g)
$$

and the functions $\phi(\xi, b)$ define the representation

$$
\left[R_{g} \phi\right](l)=\beta^{-1}(l, g) \phi(l g),
$$

where $\phi(l)=\phi(\xi, b)$ and $\beta(l, g)$ is the zeroth coordinate of $\xi g$. This pair of functions are obtained by integration of $f(x)$ over the two distinct manifolds of horospheres on $L_{3}(I)$. [We assume that the reader is familiar with the rudiments of the geometry of $L_{3}(I)$ as found for instance in Ref. 7.] Accordingly, we have

(i) Horospheres of the first kind.

$$
h(\xi)=\int f(x) \delta(|[x, \xi]|-1) d x
$$

with $d x$ the invariant measure on $L_{3}(I)$

$$
d x=\frac{d x_{1} d x_{2} d x_{3}}{\left|x_{0}\right|}
$$


Here a typical horosphere of the first kind has the equation

$$
|[x, \xi]|=1 \text {. }
$$

(ii) Horospheres of the second kind.

In this case $\phi(\xi, b)$ is obtained by integration of $f(x)$ over the isotropic line $x=b+t \xi$ according to

$$
\phi(\xi, b)=\int_{-\infty}^{\infty} f(b+t \xi) d t
$$

where

$$
[b, b]=-1, \quad[b, \xi]=[\xi, \xi]=0, \quad b_{0}=0 .
$$

The choice of integration over an isotropic line is more convenient than over the horosphere itself. We note that each horosphere of the second kind given by $[x, \xi]=0$ consists of all mutually parallel isotropic lines passing through the point $\xi$ on the cone.

$f(x)$ is given in terms of $h(\xi)$ and $\phi(\xi, b)$ by the formula

$$
\begin{aligned}
& f(x)=\frac{1}{(4 \pi)^{2}} \int h(\xi) \delta(2)(|[x, \xi]|-1) d \xi \\
& \quad+\frac{1}{(2 \pi)^{2}} \int_{0}^{\pi} \cot ^{2} \theta d \theta \int_{\Gamma} \phi(\xi, \theta) d w,
\end{aligned}
$$

where

$$
d \xi=\frac{d \xi_{1} d \xi_{2} d \xi_{3}}{\left|\xi_{0}\right|}
$$

with $\phi(\xi, \theta)$ the value of $\phi(\xi, b)$ for the isotropic line $y=b+t \xi$ lying in the $[x, y]=\cos \theta$ plane (i.e., $[x, b]=$ $\cos \theta) . \Gamma$ is a contour on the cone intersecting each generator once and the measure $d w$ is defined by

$d w=\left|\xi_{0}\right|-1\left(\xi_{1} d \xi_{2} d \xi_{3}-\xi_{2} d \xi_{1} d \xi_{3}+\xi_{3} d \xi_{1} d \xi_{2}\right)$.

In order to achieve the decomposition of $f(x)$ into irreducible parts it is necessary to expand the "Fourier components" $h(\xi)$ and $\phi(\xi, \theta)$ into homogeneous components. For $h(\xi)$ this is done exactly as for the case of $L_{3}(R)$, i.e.,

$$
\begin{aligned}
& h(\xi)=\frac{1}{2 \pi i} \int_{\delta-i \infty}^{\delta+i \infty} F(\xi ; \sigma) d \sigma \\
& F(\xi ; \sigma)=\int_{0}^{\infty} h(t \xi) t^{-\sigma-1} d t .
\end{aligned}
$$

The expansion of $\phi(\xi, \theta)$ into irreducible (homogeneous) components is achieved by Fourier analyzing $\phi(\xi, \theta)$ with respect to the angle $\theta(0 \leq \theta<\pi)$ which specifies each isotropic line in a given horosphere of the second kind. The appropriate decomposition is

$$
\phi(\xi, \theta)=\frac{1}{\pi} \sum_{n=-\infty}^{\infty} \tilde{F}(\xi ; x ; 2 n) e^{-2 i n \theta} .
$$

The "Fourier coefficients" satisfy the homogeneity condition

$$
\tilde{F}(\xi ; b ; 2 n)=\tilde{F}(\xi ; x ; 2 n) e^{-2 i n \theta} .
$$

The invariant decomposition of $f(x)$ is then

$$
\begin{aligned}
f(x) & =\frac{i}{4(2 \pi)^{3}} \int_{\delta-i \infty}^{\delta+i \infty} \sigma(\sigma+1) \int_{\Gamma} F(\xi ; \sigma)|[x, \xi]|^{-\sigma-2} d \xi d \sigma \\
& +\frac{1}{\pi^{2}} \sum_{n=1}^{\infty} n \int_{\Gamma} \tilde{F}(\xi ; b ; 2 n) e^{2} \operatorname{in}^{\infty} \delta([x, \xi]) d \xi
\end{aligned}
$$

and the inversion formulas are

$$
\begin{aligned}
& F(\xi ; \sigma)=\int f(x)|[x, \xi]| \sigma d x, \\
& \tilde{F}(\xi ; b ; 2 n)=\int f(x) e^{-2} \operatorname{in} \theta \delta([x, \xi]) d x .
\end{aligned}
$$

Group theoretically the "Fourier coefficients" in (1.14) transform according to the irreducible representations (IRs) of $S O(3,1)$ as follows:

(i) $F(\xi ; \sigma)$ transform according to the IRs

$$
c=\sigma+1=\delta+1+i \rho, \quad(-\infty<\rho<\infty), \quad k_{0}=0,
$$

where $\left[c, k_{0}\right]$ labels each IR of $S O(3,1)$. (This is the notation due to Naimark ${ }^{13}$ that we are using here.) We obtain the unitary case (i.e., the principal series) when $\delta=-1$.

(ii) $\bar{F}(\xi ; b ; 2 n)$ transform according to the UIRs $S O(3,1)$

$$
c=0, \quad k_{0}=2 n, \quad n=1,2,3, \cdots .
$$

\section{THE HOROSPHERICAL OR Ho SYSTEM EXPANSION ON $L_{3}(/)$}

The Ho system 4-vector $x$ on the single sheet hyperboloid $H_{1}$ is given by

$$
\begin{aligned}
& x=\left(\frac{1}{2}\left[-e^{-a}+\left(1+r^{2}\right) e^{a}\right], r e^{a} \cos \phi, r e^{a} \sin \phi,\right. \\
& \quad \frac{1}{2}\left[-e^{-a}+\left(r^{2}-1\right) e^{a}\right], \\
& -\infty<a<\infty, \quad 0 \leq r<\infty, \quad 0 \leq \phi<2 \pi .
\end{aligned}
$$

This parametrization covers the $x_{0}-x_{3} \geq 0$ half of the $[x, x]=-1$ hyperboloid and so covers all of $L_{3}(I)$.

For the Ho system expansion the contour $\Gamma$ is taken to be

$$
\xi_{0}-\xi_{3}=2
$$

and $\xi$ is parametrized according to

$$
\begin{aligned}
\xi= & \left(1+u^{2}+v^{2}, 2 u, 2 v,-1+u^{2}+v^{2}\right), \\
& -\infty<u, v<\infty .
\end{aligned}
$$

$F(\xi ; \sigma)$ is expanded in a double Fourier series according to

$$
F(\xi ; \sigma)=\int_{-\infty}^{\infty} \int_{-\infty}^{\infty} a_{\lambda \mu}(\sigma) e^{i \lambda u} e^{i \mu v} d \lambda d \mu
$$

and the measure on the cone is

$$
d \xi=4 d u d v .
$$

Taking $x=$ (sha, $0,0,-$ cha) the continuous spectrum part of expansion (1.14) then reduces to the calculation of the integral

$I_{\lambda \mu}^{\circ}(a)=\int_{-\infty}^{\infty} d u \int_{-\infty}^{\infty} d v \mid e^{a}\left(u^{2}+v^{2}\right)-e^{-a \mid-\sigma-2} e^{i \lambda u} e^{i \mu v}$.

This integral can be calculated by using the identity

$$
|t|^{\beta}=t_{+}^{\beta}+t_{-}^{B}
$$

as well as the known Fourier transforms in two dimensions of the functions $\left(b^{2}-u^{2}-v^{2}\right)_{t}^{-o-2}$ which are 
given by

$$
\begin{aligned}
\text { F.T. } & {\left[\frac{\left(b^{2}-u^{2}-v^{2}\right)^{-\sigma-2}}{\Gamma(-\sigma-1)}\right] } \\
& =-i(2 b)^{-\sigma-1}\left[\frac{K_{-\sigma-1}(b(Q-i 0))}{(Q-i 0)^{-(\sigma+1)}}-\frac{K_{-\sigma-1}(b(Q+i 0))}{(Q+i 0)^{-(\sigma+1)}}\right],
\end{aligned}
$$

$$
\begin{aligned}
& \text { F.T. }\left[\frac{\left(b^{2}-u^{2}-v^{2}\right)_{+}^{-\sigma-2}}{\Gamma(-\sigma-1)}\right]=-i(2 b)^{-\sigma-1} \\
& \times\left[e^{i \pi(\sigma+1)} \frac{K_{-\sigma-1}(b(Q-i 0))}{(Q-i 0)^{-(\sigma+1)}}-e^{-i \pi(\sigma+1)} \frac{K_{-\sigma-1}(b(Q+i 0))}{(Q+i 0)^{-(\alpha+1)}}\right] \text {, }
\end{aligned}
$$

where $Q=-\lambda^{2}-\mu^{2}$.

These formulas are special cases of the general formulas for the Fourier transforms in $n$ dimensions of the generalized functions $\left(b^{2}+P\right)^{\lambda}(\lambda \neq$ integer $)$ as given by Gel'fand and Shilov. ${ }^{14}$ (Note: $P$ is a general quadratic form in the $n$ Cartesian coordinate variables expressed in canonical or diagonal form.) $I_{\lambda \mu}^{\sigma}(a)$ is then found to be

$I_{\lambda \mu}^{\circ}(a)=i \pi\left(\frac{\chi}{2}\right)^{\sigma+1} \Gamma(-\sigma-1) e^{-a}\left[J_{0+1}\left(e^{-a} \chi\right)+J_{-\sigma-1}\left(e^{-a}\right)\right]$,

where $x=\left(\lambda^{2}+\mu^{2}\right)^{1 / 2}$.

For the discrete part of expansion $(1.14), \tilde{F}(\xi ; 2 n)$ is expanded according to

$$
\begin{aligned}
& \tilde{F}(\xi ; 2 n)=\sum_{m=-\infty}^{\infty} \int_{0}^{\infty} \chi^{d}{ }_{X} a_{m}(\chi ; 2 n) J_{2 n-m}(\chi p) e^{i m \psi}, \\
& \text { where } u=p \cos \psi, v=p \sin \psi
\end{aligned}
$$

The evaluation of the discrete part of $(1.14)$ then requires the calculation of

$$
I_{x}(2 n)=\int J_{2 n-m}(x p) e^{i m \psi} \delta([x, \xi]) d \xi .
$$

This integral is readily calculated, using the identity

$$
\delta\left(a^{2}-x^{2}\right)=\frac{1}{2 a}[\delta(a+x)+\delta(a-x)],
$$

to be

$$
I_{\chi}(2 n)=4 \pi e^{-a} J_{2 n}\left(\chi e^{-a}\right) J_{m}\left(\chi^{r}\right) e^{i m \phi} .
$$

The $H o$ system expansion on $L_{3}(I)$ is then

$$
\begin{aligned}
f(x)= & \sum_{m=-\infty}^{\infty} \int_{0}^{\infty} \chi^{d} \chi e^{-a}\left(\frac{-1}{8(2 \pi)^{2}} \int_{\delta-i \infty}^{\delta+i \infty}\right. \\
& \times \sigma(\sigma+1) a_{m}(\chi, \sigma) \Gamma(-\sigma-1) \\
& \times\left[J_{\sigma+1}\left(e^{-a} \chi\right)+J_{-\sigma-1}\left(e^{-a} \chi\right)\right] d \sigma \\
& \left.+\frac{4}{\pi} \sum_{n=1}^{\infty} n a_{m}(x, 2 n) J_{2 n}\left(\chi e^{-a}\right)\right) J_{m}\left(\chi^{r}\right) e^{i m \phi} .
\end{aligned}
$$

For the continuous part of $(1.14)$ we have changed the expansion of $F(\xi ; \sigma)$ to polar coordinates and used the identity

$$
e^{i \times r \cos (\phi-\theta)}=\sum_{m=-\infty}^{\infty} i^{m} J_{m}\left(\chi^{r}\right) e^{i m(\phi-\theta)},
$$

where $\tan \theta=\lambda / \mu$.

$$
a_{m}(\mathrm{x} ; \sigma) \text { is then given by }
$$

$$
a_{m}(x ; \sigma)=i^{m} \int_{0}^{2 \pi} a_{\lambda \mu}(\sigma) e^{-i m \theta} d \theta
$$

The inversion formulas of (2.15) are

$$
\begin{aligned}
& a_{m}(\chi ; \sigma)=\frac{i}{4 \pi} \Gamma(\sigma+1) \int f(x) e^{-a}\left[J_{\sigma+1}\left(e^{-a} \chi\right)\right. \\
& \left.+J_{-\sigma-1}\left(e^{-a} \chi\right)\right] J_{m}(\chi \gamma) e^{-i m \phi} d x, \\
& a_{m}(\chi ; 2 n)=\frac{1}{2} \int f(x) e^{-a} J_{2 n}\left(\chi e^{-a}\right) J_{m}\left(\chi^{\gamma}\right) e^{-i m \phi} d x,
\end{aligned}
$$

where

$$
d x=e^{2 a} d a r d r d \phi .
$$

For the principal series $\sigma=-1+i \rho$ the continuous part of $(3.15)$ is an expansion in terms of the functions

$$
\Psi_{\rho \chi m}^{H o}(a, r, \phi)=e^{-a} \tilde{J}_{i \rho}\left(\chi e^{-a}\right) J_{m}\left(\chi^{r}\right) e^{i m \phi}
$$

which satisfy the orthogonality relations

$$
\begin{aligned}
& \int \Psi_{\rho \mathrm{X} m}^{H o}(a, r, \phi) \Psi_{\overline{\bar{p}_{\overline{\mathrm{X}}} \bar{m}}}^{\overline{o_{\bar{m}}}(a, r, \phi) d x} \\
& =\frac{2 \operatorname{sh} \pi \rho}{\mathrm{x} \rho} \delta(\rho-\bar{\rho}) \delta(\mathrm{x}-\bar{\chi}) \delta_{m \bar{m}},
\end{aligned}
$$

where we have put

$$
\tilde{J}_{i \rho}(x)=J_{i \rho}(x)+J_{-i \rho}(x) \text {. }
$$

We observe that the $a$ dependant part of (2.22) reproduces the completeness relation for the Titchmarsh integral transform, 15 i.e.,

$$
\int_{0}^{\infty} \tilde{J}_{i \rho}(x) \tilde{J}_{i \bar{\rho}}(x) x^{-1} d x=\frac{2 \operatorname{sh} \pi \rho}{\rho} \delta(\rho-\bar{\rho})
$$

We also note that for the discrete spectrum expansion functions we have the orthogonality relation

$$
\int_{0}^{\infty} J_{2 n}(x) J_{2 m}(x) x^{-1} d x=\frac{1}{4 n} \delta_{n m} .
$$

This is just a special case of the formula

$$
\int_{0}^{\infty} J_{\mu}(x) J_{\nu}(x) x^{-1} d x=\frac{2 \sin \frac{1}{2} \pi(\nu-\mu)}{\pi\left(\nu^{2}-\mu^{2}\right)} .
$$

\section{CONCLUDING REMARKS}

We have given here an expansion of a function $f(x) \in L_{3}(I)$ in a coordinate system which is an alternative to the canonical or $S$ system, viz., the Ho system. The parametrization of $x$ we used is obtained from the corresponding coordinate system vector on $\mathrm{H}_{2}$ via the analytic continuation $a \rightarrow a+i \pi / 2$. This example illustrates not only the application of the analysis of Gel'fand and Graev in obtaining explicit expansion formulas but also that the group reduction parametrizations of $x \in H_{2}$ when continued in the manner above do not always cover 
all of $H_{1}$. The $S$ system is the only one that covers all of $H_{1}$. It should be mentioned here that the expansion functions used for $\tilde{F}(\xi, 2 n)$ in (2.11) are the natural ones in the sense that they are the basis functions for the UIR $\{o, 2 n\}$ of $S O(3,1)$ when realized in a $H o$ system basis in the space of square-integrable functions in the plane.

In the future we intend to study all possible coordinate systems on $H_{1}$ which cover at least all of $L_{3}(I)$ and for which the angular part of the Laplacian $\Delta_{L}$ admits a separation of variables.

'For perhaps the most complete set of references see P. Winternitz "Two variable expansions based on the Lorentz and conformal groups," talk presented at Symposium on the de Sitter and conformal groups, Boulder, Colorado, 1970 and references contained therein. ${ }^{2}$ J. S. Zmuidzinas, J. Math. Phys. 7, 764 (1966).

${ }^{3}$ N. Limic, J. Niederle, and R. Raczka, J. Math. Phys. 8, 1079 (1967).

${ }^{4}$ N. Ya. Vilenkin and Ya. A. Smorodinski, Zh. Eksp. Teor. Fiz.
46, 1793 (1964) [Sov. Phys.-JETP 19, 1209 (1964)]

${ }^{5}$ M. A. Liberman and A. A. Makarov, Sov. J. Nucl. Phys. 9, 766 (1969).

${ }^{6}$ E. G. Kalnins, Ph.D. thesis, University of Western Ontario (1972)

${ }^{7}$ I. M. Gel'fand, M. I. Graev, and N. Ya. Vilenkin, Generalized Functions (Academic, New York, 1966), Vol. 5

${ }^{8}$ G. I. Kuznetsov and Ya. A. Smorodinski, Sov. J. Nucl. Phys. 3, 275 (1966)

${ }^{9}$ I. A. Verdiev, Sov. J. Nucl. Phys. 10, 726 (1970).

${ }^{10}$ E. C. Titchmarsh, Eigenfunction Expansions (Clarendon Press, Oxford, 1962), Part I

${ }^{11}$ S. J. Chang and L. O'Raifeartaigh, J. Math. Phys. 10, 21 (1969).

${ }^{12}$ P. Winternitz, Ya. A. Smorodinski, and M. B. Sheftel, Sov. J. Nucl Phys. 7, 785 (1968).

${ }^{13}$ M. A. Naimark, Linear Representations of the Lorentz Group (Pergamon, New York, 1964).

${ }^{14}$ I. M. Gel'fand and G. E. Shilov, Generalized Functions (Academic, New York, 1964), Vol. 1.

${ }^{15}$ See in this connection Eq. 5, p. 150 of Formulas and Theorems for the Special Functions of Mathematical Physics by W. Magnus, F. Oberhettinger, and R. P. Soni (Springer-Verlag, New York, 1966). 